\title{
Häusliche Gewalt - was können Ärztinnen und Ärzte tun?
}

Martha Weingartner,

Brida von Castelberg

* Das Projekt «Häusliche Gewalt wahrnehmen - intervenieren» sowie die Leitlinien zum Vorgehen bei häuslicher Gewalt sind im Handbuch «Häusliche Gewalt erkennen und richtig reagieren» [1] ausführlich dargestellt.

Korrespondenzen:

Martha Weingartner

Fachstelle für Gleichstellung

Stadt Zürich

Ausstellungsstrasse 88

CH-8005 Zürich

Tel. 0444471779

martha.weingartner@zuerich.ch

www.stadt-zuerich.ch/gleichstellung

Dr. med. Brida von Castelberg

Frauenklinik Maternité

Stadtspital Triemli Zürich

Birmensdorferstrasse 501

CH-8063 Zürich

Tel. 0444661111

brida.voncastelberg@triemli.stzh.ch

www.stadtzuerich.ch/internet/ triemli/home/kliniken/maternite frauenklinik.html
Seit einigen Jahren wird «häusliche Gewalt» zunehmend in Fachkreisen und in der Öffentlichkeit diskutiert. Behörden und Institutionen (Polizei, Strafverfolgungsbehörden, Beratungsstellen) haben Massnahmen entwickelt, um ihre Interventionen bei häuslicher Gewalt zu verbessern. Gesetze wurden angepasst oder neu eingeführt, um wirksamer gegen häusliche Gewalt vorgehen und die Opfer besser schützen zu können. Eine wichtige Rolle - insbesondere bei der Früherkennung von häuslicher Gewalt - haben Fachleute im Gesundheitsbereich.

\section{Ärztinnen und Ärzte sind wichtige Anlaufstellen}

Viele Gewaltopfer - in den meisten Fällen Frauen, aber auch Männer können von häuslicher Gewalt betroffen sein - wenden sich zunächst nicht an eine Beratungsstelle und rufen auch nicht die Polizei zu Hilfe. Aber sie konsultieren aufgrund von Verletzungen oder gesundheitlichen Beschwerden eine Ärztin oder einen Arzt oder suchen eine Notfallstation auf.

Für die betroffenen Berufsgruppen ist der Umgang mit häuslicher Gewalt nicht einfach, denn selten liegt das Thema klar auf dem Tisch. Angst, in die Privatsphäre einzudringen, Zeitdruck und Unsicherheit bezüglich möglicher Reaktionen verhindern ein aktives Ansprechen des Problems. Auch den Betroffenen fällt es meistens sehr schwer, über ihre Erfahrungen zu sprechen. Häusliche Gewalt ist noch immer ein Tabuthema, das mit Scham- und Schuldgefühlen verbunden ist.

\section{Pionierprojekt an der Frauenklinik Maternité in Zürich}

In Kliniken und Spitälern ist es sinnvoll, eine sogenannte «Policy» zu entwickeln, also ein von der Leitung her gestütztes Vorgehen, das den Umgang mit «häuslicher Gewalt» festlegt. Damit wird gewährleistet, dass das Handeln bei häuslicher Gewalt einheitlich und kompetent ist. Die Mitarbeiterinnen und Mitarbeiter bekommen Handlungssicherheit und sind besser geschützt vor Überforderung, da Fälle von häuslicher Gewalt sehr belasten können.

Die Frauenklinik Maternité (Stadtspital Triemli, Zürich) hat in Zusammenarbeit mit der Fach-

\section{Violence domestique - que peut faire le médecin?}

Une étude menée à la maternité de l'Hôpital municipal de Triemli à Zurich a montré que la violence domestique subie par des patientes a considérablement augmenté. Dix femmes sur les 1779 interrogées ont vécu, l'année de l'enquête, un acte de violence domestique intenté par une personne de l'entourage, le plus souvent par leur partenaire. Le médecin joue un rôle important dans la reconnaissance précoce des actes de violence. A cet effet, il est essentiel qu'il prenne en considération la violence domestique comme étant une cause potentielle de lésions ou de troubles de la santé. II devrait interpeller plus souvent ses patientes sur leurs éventuelles expériences de violence domestique. Même s'il ne peut résoudre lui-même le problème, le fait d'en parler encourage les patientes à chercher de I'aide. Une connaissance des fondements de la violence domestique et des offres d'assistance locale constituent un atout indispensable pour le praticien. II devrait aussi avoir la compétence nécessaire pour réagir de manière adéquate dans des cas concrets. Les cliniques et les hôpitaux sont invités à mettre au point une stratégie commune pour approcher le phénomène et enseigner à leurs collaborateurs comment réagir.

stelle für Gleichstellung der Stadt Zürich eine solche «Policy» entwickelt. Von 2002 bis 2006 führte sie das Projekt «Häusliche Gewalt - wahrnehmen - intervenieren ${ }^{*}$ durch. Das Projekt beinhaltete eine Befragung der Mitarbeitenden und eine repräsentative Patientinnenbefragung, die Entwicklung von Leitlinien und Schulungsprogramme. Die «Leitlinien zum Vorgehen bei häuslicher Gewalt» beinhalten unter anderem ein sogenanntes Screening. Das heisst, dass die Patientinnen im Rahmen der Anamnese nach häuslicher Gewalt gefragt werden. Die Erfah- 


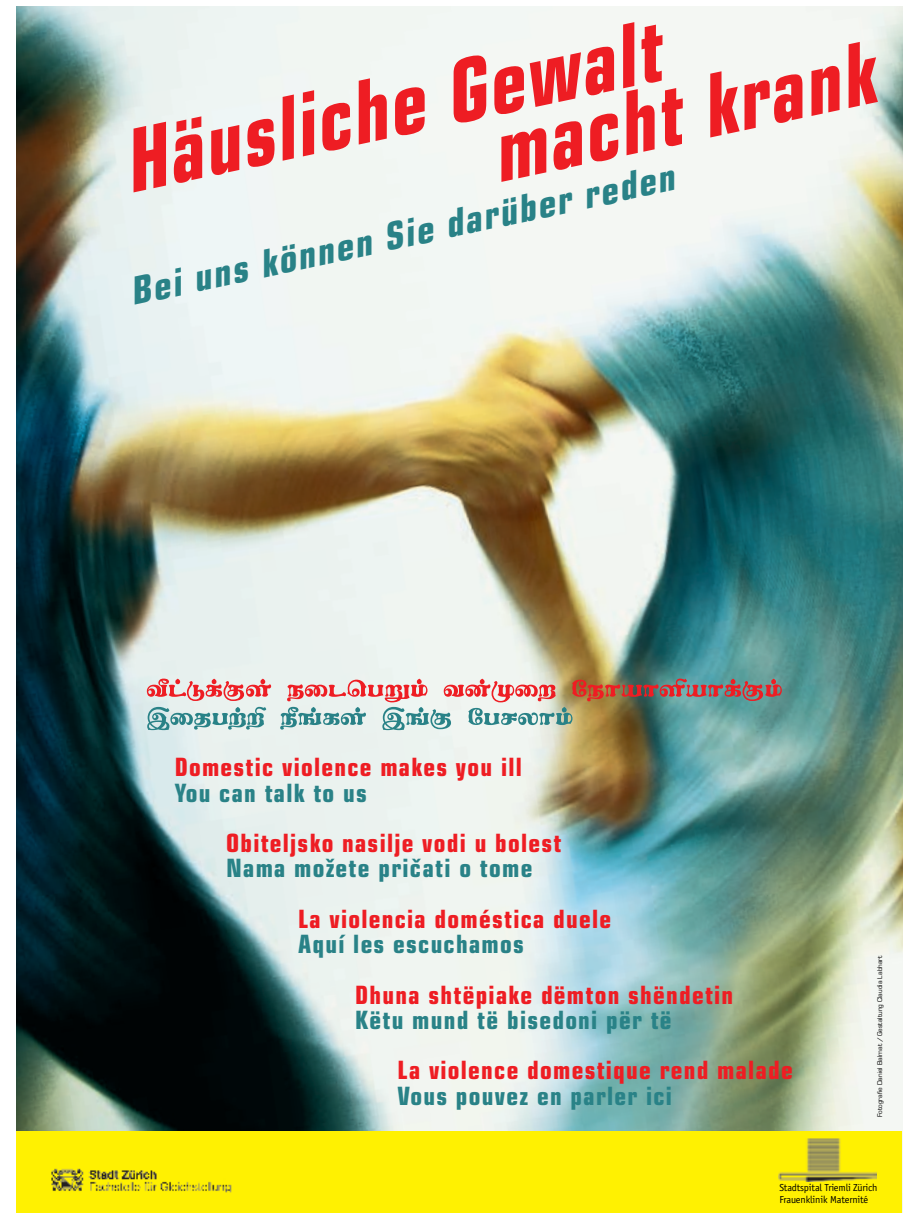

rungen mit dem Screening sind positiv. Die Patientinnen begrüssen es, dass häusliche Gewalt enttabuisiert wird. Es ist ein Signal, dass häusliche Gewalt ernst genommen wird, dass das Thema sein darf und dass es Hilfe und Unterstützung gibt.

Als weitere Massnahmen wurden ein mehrsprachiges Booklet mit Beratungsadressen erarbeitet und ein Plakat gestaltet, das ebenfalls in mehreren Sprachen sagt: «Häusliche Gewalt macht krank - Hier können Sie darüber reden.» (Booklet und Plakat sind bei der Fachstelle für Gleichstellung gratis zu beziehen.)

\section{Häusliche Gewalt kommt häufig vor und ist ein Gesundheitsproblem}

Die an der Frauenklinik Maternité durchgeführte Studie [2] hat ergeben, dass jede 10. von 1779 befragten Frauen im Jahr vor der Befragung häusliche Gewalt durch eine nahestehende Person erlebt hat. Am häufigsten durch den aktuellen Beziehungspartner. Die Betroffenheit blieb in etwa gleich, wenn die Zahlen nach unterschiedlichen Merkmalen wie etwa Bildung, Nationalität oder Einkommen ausgewertet wurden. Zwischen erlebter Gewalt und der gesundheitlichen
Situation der Patientinnen konnte ein starker Zusammenhang festgestellt werden. Je stärker die Frauen im Laufe ihres Lebens Gewalt erlitten hatten, je schlechter war ihr Gesundheitszustand.

Gemäss der Weltgesundheitsorganisation WHO stellt häusliche Gewalt weltweit das höchste Gesundheitsrisiko für Frauen dar [3]. Sie rangiert bei Frauen vor Krebserkrankungen und Verkehrsunfällen.

\section{Beziehungsproblem oder «häusliche Gewalt»?}

Häusliche Gewalt wird oft verwechselt oder gleichgesetzt mit Konflikten und Streit. Dies hat für die Opfer von häuslicher Gewalt fatale Folgen. Die Gewalt und ihre Auswirkungen werden nicht wahrgenommen und verharmlost. Die gewalttätige Person erfährt keine Konsequenzen. Deshalb ist es wichtig zu erkennen, ob es sich um einen Streit zwischen zwei gleichberechtigten Partnern handelt oder um häusliche Gewalt. Häusliche Gewalt ist gekennzeichnet durch Machtausübung und Kontrolle und führt $\mathrm{zu}$ einem asymmetrischen Beziehungsverhältnis. Bereits bestehende Abhängigkeiten in der Beziehung (z.B. ökonomische, aufenthaltsrechtliche) werden ausgenutzt, das Opfer erlebt einen Autonomieverlust.

Häusliche Gewalt ist in den meisten Fällen kein einmaliges Vorkommnis, sondern wiederholt sich und nimmt mit der Zeit an Intensität zu. Es werden verschiedene Formen von körperlicher, psychischer und sexueller Gewalt angewendet, die einzelnen Gewaltformen gehen ineinander über. Es handelt sich also nicht um sporadische emotionale Ausbrüche, sondern um ein System von Kontrolle und Macht, das die Gefahr der Gewalteskalation beinhaltet. Phasen des Spannungsaufbaus, akute Gewalthandlungen und Phasen der Versöhnung und Ruhe wechseln sich ab. Auf Gewaltakte folgen die Reue und die Zuwendung des Gewalttäters, verbunden mit Versprechungen, dass so etwas nie mehr vorkommt. Gewaltbetroffene Frauen sind immer wieder bereit, auf diese Versprechungen einzugehen. In der Hoffnung, dass sich der Partner doch noch ändere, versuchen sie mittels Anpassungsstrategien weitere Gewalteskalationen zu vermeiden. Doch sie können nicht verhindern, dass es wieder zu einem Spannungsaufbau und zur Gewaltausübung kommt. Die Opfer werden immer schwächer und fühlen sich mitschuldig, da es ihnen nicht gelingt, die Spirale der Gewalt zu durchbrechen. In den meisten Fällen braucht es mehrere Anläufe und die Hilfe von aussen, bis dies gelingt. 


\section{Trennung löst das Gewaltproblem häufig nicht}

Häusliche Gewalt findet zwischen nahestehenden Personen statt, also zwischen Menschen, die durch Gefühle, Pflichten und Abhängigkeiten miteinander verbunden sind. Helfende können häufig nicht verstehen, warum gewaltbetroffene Frauen ihren Partner nicht verlassen oder immer wieder zu ihm zurückkehren. Viele Frauen verhalten sich ambivalent ihren gewalttätigen Partnern gegenüber. Trotzdem will jede Frau, dass die Gewalt aufhört. Wichtig zu wissen ist, dass mit einer Trennung das Gewaltproblem häufig noch nicht gelöst ist. Vielmehr ist bei Trennungsabsichten besondere Vorsicht geboten, denn die meisten Tötungsdelikte bei häuslicher Gewalt geschehen in der Phase von Trennung und Scheidung.

\section{Anzeichen von häuslicher Gewalt erkennen}

Die gesundheitlichen Folgen und Auswirkungen von häuslicher Gewalt sind vielfältig und können gravierend sein. Nicht nur schwere körperliche Gewalt führt zu Beeinträchtigungen der Gesundheit, auch Drohungen und ein damit verbundenes Leben in ständiger Angst machen krank.

Ärztinnen und Ärzte können in aller Regel die Gewalt nicht beenden, ihre Diagnostik und Beratung bestimmen aber wesentlich das weitere Geschehen. Der erste Schritt ist das Inbetrachtziehen von häuslicher Gewalt als mögliche Ursache von Verletzungen und gesundheitlichen Beschwerden. Diese reichen von sichtbaren Verletzungen bis hin zu diffusen somatischen, nicht selten schon chronischen Beschwerdebildern. Körperliche Misshandlungen wie Schläge, Verbrennungen, Bisse, Würgen usw. hinterlassen oft sichtbare Spuren. Folgen von psychischer und/oder sexueller Gewalt sind für Aussenstehende nicht sofort sichtbar oder überhaupt als solche zu erkennen. Eine eindeutige Verbindung von der Ursache der Verletzung und dem Erscheinungsbild fehlt häufig. Dazu kommt, dass Betroffene selten von sich aus die Ursache ihrer Verletzungen und Beschwerden ansprechen. Diverse Studien, so auch die Untersuchung an der Frauenklinik Maternité, zeigen jedoch, dass es die meisten Frauen, die sich in ärztliche Behandlung begeben, befürworten, auf erlittene Gewalt angesprochen zu werden. Ein Patentrezept zum Erkennen von häuslicher Gewalt gibt es nicht. Die sogenannten «Red Flags» (s. Kasten) können bei der Einschätzung, ob häusliche Gewalt vorliegt, behilflich sein.

\section{Häusliche Gewalt ansprechen}

Es ist also hilfreich, häusliche Gewalt als mögliche Ursache der Verletzungen direkt anzusprechen. Damit wird der Patientin signalisiert, dass häusliche Gewalt Thema sein darf und dass dazu Gesprächsbereitschaft vorhanden ist. Auch der Hinweis auf die Schweigepflicht, mit der Präzisierung, dass niemand, auch nicht der Partner oder die Familie, etwas davon erfährt, kann unterstützend sein, insbesondere bei Migrantinnen, die die Bedeutung der Schweigepflicht vielleicht nicht kennen. Wenn die Patientin nicht auf das Gesprächsangebot eingehen möchte, ist es wichtig, ihr anzubieten, dass sie jederzeit darauf zurückkommen kann.

Bei der Gesprächsführung sind einige Grundregeln zu beachten, die im folgenden kurz zusammengefasst sind:

- vermutete Gewalt nicht in Anwesenheit des Partners ansprechen;

\section{Red Flags}

1. Chronische Beschwerden, die keine offensichtliche physische Ursache haben

2. Verletzungen, die nicht mit der Erklärung, wie sie entstanden sind, übereinstimmen

3. Verschiedene Verletzungen in unterschiedlichen Heilungsstadien

4. Partner, der übermässig aufmerksam ist, kontrolliert und nicht von der Seite der Frau weichen will

5. Physische Verletzungen während der Schwangerschaft

6. Spätes Beginnen der Schwangerschaftsvorsorge

7. Häufige Fehlgeburten

8. Häufige Suizidversuche und -gedanken

9. Verzögerung zwischen Zeitpunkt der Verletzung und Aufsuchen der Behandlung

10. Chronische Darmstörung (Reizdarm)

11. Chronische Beckenschmerzen

Das gleichzeitige Auftreten mehrer Red Flags sollte hohe Aufmerksamkeit auslösen. Es weist auf eine hohe Wahrscheinlichkeit von Gewalterfahrungen hin. 
- das Erlebte nicht in Frage stellen;

- nicht urteilen, auch nicht über die gewalttätige Person;

- Schutz und Sicherheit beachten;

- Entscheide der Betroffenen respektieren.

Auch wenn die Frau vielleicht im Moment (noch) nicht bereit ist, ein Beratungsangebot anzunehmen, so macht sie vielleicht zu einem späteren Zeitpunkt davon Gebrauch. Zum Überwinden der Schwellenangst kann es manchmal helfen, ihr anzubieten, den Erstkontakt zu einer Beratungsstelle herzustellen.

Vorschnelle Ratschläge sind wenig hilfreich, ebensowenig wie Massnahmen, die über den Kopf der Patientin hinweg eingeleitet werden. Die Frau ist zwar auf Hilfe und Unterstützung angewiesen, aber es ist wichtig, dass sie die Entscheidungen selber treffen kann. Ohne das Einverständnis der Patientin, initiativ zu werden, z.B. durch die Information der Polizei oder die Konfrontation mit dem gewalttätigen Mann, kann die Betroffene zusätzlich gefährdet werden.

Wenig sinnvoll ist es, in Fällen von häuslicher Gewalt eine Paarberatung zu empfehlen. Das bestehende Machtgefälle in der Beziehung verunmöglicht das offene Gespräch und kann eine gewaltbetroffene Person zusätzlich unter Druck setzen. Sinnvoller ist es, wenn sich der gewalttätige Mann z.B. in einem «mannebüro» [4] oder einer Hotline für gewalttätige Männer unterstützen lässt und die gewaltbetroffene Frau bei einer Opferberatungsstelle [5] Hilfe bekommt.

\section{Wichtigstes Ziel: Schutz und Sicherheit}

Die Sicherheit der Patientin muss oberstes Gebot sein. Die Gefährdungslage muss mit der Frau besprochen werden, und es ist zu klären, ob sie nach Hause zurückkehren bzw. zu Hause bleiben kann und will. Wichtig ist dabei, auch die Situation allfälliger Kinder einzubeziehen. Wenn weiterhin eine Gefährdung besteht, ist möglicherweise ein Eintritt in ein Frauenhaus [6] angezeigt.

Bei einem stationären Aufenthalt muss geklärt werden, wie mit gewalttätigen Angehörigen umgegangen werden kann und ob es Schutzmassnahmen braucht.

Falls Sie als Ärztin/Arzt die Gefährdung einer Patientin höher einschätzen als die Patientin selbst, gilt es, notwendige Schutzmassnahmen genau zu prüfen. Es gibt Situationen, in denen Sie gegen den Willen der Patientin z.B. die Polizei einschalten müssen. Solche Situationen sind heikel, und es empfiehlt sich in jedem Fall, sich mit einer weiteren Fachperson abzusprechen, z.B. einer Mitarbeiterin einer Opferberatungsstelle.

\section{Untersuchen und Dokumentieren}

Eine gründliche Untersuchung und sorgfältige Dokumentation sind in jedem Fall von häuslicher Gewalt zwingend notwendig. Zwar benötigen gewaltbetroffene Frauen zum Zeitpunkt der Konsultation sehr oft keine Dokumentation. Eine solche kann aber später von entscheidender Bedeutung sein, insbesondere in einem Strafund/oder Trennungsverfahren, aber auch im Zusammenhang mit aufenthaltsrechtlichen Fragen oder in bezug auf Leistungen nach Opferhilfegesetz. Die meisten Betroffenen können die erlittene Gewalt nicht nachweisen, deshalb ist der Nachweis durch eine medizinische Dokumentation so wichtig.

Eine Dokumentation [7] sollte die folgenden Angaben enthalten:

- Schilderung der Gewalttat (detailgetreu festhalten, in den Worten der Patientin);

- Angaben zu früheren Misshandlungen (in den Worten der Patientin festhalten);

- genaue Beschreibung der Verletzungen und des körperlichen Befundes;

- Diagnose oder Verdachtsdiagnose;

- Bewertung des Befundes im Kontext der Patientin (ist der Befund mit den Schilderungen der Patientin zu «vereinbaren»?);

- Fotodokumentation (mit Massstab);

- Angabe der weiterführenden Massnahmen.

Für Untersuchungen bei bestehenden Verdachtsmomenten (z.B., ob eine Verletzung lebensbedrohlich war) ist oft forensisches Spezialwissen notwendig. Die rechtsmedizinischen Institute der Kantone Zürich, Bern, Basel und St. Gallen haben einen 24-Stunden-Betrieb und stehen Ärztinnen und Ärzten konsiliarisch zur Verfügung.

\section{Rechtliche Grundlagen}

Seit dem 1. April 2004 ist «häusliche Gewalt» ein Offizialdelikt. Wenn Behörden von einer möglichen Straftat erfahren, müssen sie von Amtes wegen ein Strafverfahren einleiten. Häufig müssen Strafverfahren aber mangels Beweisen, z.B. weil das Opfer nicht aussagen will, wieder eingestellt werden. In einigen Kantonen hat die Polizei die Möglichkeit, bei häuslicher Gewalt Massnahmen zum unmittelbaren, kurzfristigen Schutz auszusprechen. Es handelt sich dabei um sogenannte Wegweisungen der gefährdenden Person, verbunden mit Betret- und Kontaktverboten.

In Kliniken oder Arztpraxen arbeitende Fachpersonen unterstehen grundsätzlich der ärztlichen Schweigepflicht. Eine Meldepflicht besteht bei verdächtigen und aussergewöhnlichen Todesfällen, die Folge eines Delikts, eines Unfalls oder 
Suizids sein können. Einige kantonale Gesundheitsgesetze sehen vor, dass Ärztinnen und Ärzte das Recht-aber nicht die Pflicht - haben, bei Verdacht auf Straftaten gegen Leib und Leben eine polizeiliche Anzeige zu machen, ohne dass eine Entbindung von der Schweigepflicht vorliegt. Besteht der Verdacht, dass Minderjährige Opfer von Misshandlungen sind, können unter dem Amts- oder Berufsgeheimnis stehende Personen ohne Einwilligung der Eltern oder des minderjährigen Kindes eine Anzeige bei der Vormundschaftsbehörde erstatten.

\section{Häusliche Gewalt verhindern durch Fachwissen und Kooperation}

Häusliche Gewalt hat rechtliche, soziale und gesundheitliche Dimensionen. Wirksame Prävention und Intervention ist auf das Zusammenspiel verschiedener Fachdisziplinen angewiesen. Wichtige Voraussetzungen dafür sind Fachwissen über die Hintergründe von häuslicher Gewalt, Handlungskompetenz sowie Kenntnisse über das lokale Hilfsangebot. Dieses Wissen muss in der Aus- und Weiterbildung vermittelt werden. In verschiedenen Kantonen gibt es Bildungsangebote für Fachleute aus dem Gesundheitsbereich. Sensibilisierte und informierte Ärztinnen und Ärzte leisten einen unverzichtbaren Beitrag zur Früherkennung und Prävention von häuslicher
Gewalt. Dieser Beitrag ist um so wichtiger, wenn die Auswirkungen häuslicher Gewalt auf die Kinder mitbedacht werden. Das Erleben von häuslicher Gewalt in der Kindheit ist einer der wichtigsten Risikofaktoren für eigene Gewalthandlungen oder Opfererfahrungen als Erwachsene.

\section{Literatur}

1 Fachstelle für Gleichstellung Stadt Zürich, Frauenklinik Maternité Stadtspital Triemli Zürich, Verein Inselhof Triemli Zürich (Hrsg.). Häusliche Gewalt erkennen und richtig reagieren. Handbuch für Medizin, Pflege und Beratung. Bern: Hans Huber; 2007.

2 Gloor D, Meier H. Frauen, Gesundheit und Gewalt im sozialen Nahraum. Repräsentativbefragung bei Patientinnen der Maternité Inselhof Triemli, Klinik für Geburtshilfe und Gynäkologie. Bern: Edition Soziothek; 2004.

3 WHO. Weltbericht Gewalt und Gesundheit. Genf: WHO; 2003.

4 www.mannebuero.ch.

5 Die kantonalen Opferhilfeberatungsstellen bieten vertrauliche und kostenlose Beratung an. Adressen: www.opferhilfe-schweiz.ch.

6 Adressen und Information über freie Plätze: www.frauenhaus-schweiz.ch.

7 Eine Mustervorlage für einen Dokumentationsbogen findet sich im Handbuch «Häusliche Gewalt erkennen und richtig reagieren» [1]. 